\title{
Long-Term Landau-Fluid Simulation of Alfvén Eigenmode Instabilities $^{*}$ )
}

\author{
Donald A. SPONG \\ Oak Ridge National Laboratory, Oak Ridge, Tennessee, USA
}

(Received 21 November 2013 / Accepted 28 March 2014)

\begin{abstract}
An energetic particle Landau-fluid closure model (TAEFL) is applied to a case where both RSAE (Reversed Shear Alfvén Eigenmode) and TAE (Toroidal Alfvén Eigenmode) modes are destabilized by beam ions. The nonlinear evolution of the coupled modes are followed for about $10^{4}$ Alfvén times under the simplifying assumptions that a density source is present that exactly balances the quasi-linear fast ion profile changes driven by the instabilities. Saturation is achieved via self-regulation/organization through zonal flows and currents. A quasi-stationary nonlinear state persists, which is characterized by repetitive predator-prey phenomena. As the mode grows, it drives zonal flows/currents, these quench its amplitude until the linear mode structure sets in again and grows, driving further zonal flows/currents, etc. This capability of following nonlinear fast ion-driven instabilities over longer time intervals (achieved here by using a higher order time-stepping method) is an important requirement for future integrated models of burning plasmas.
\end{abstract}

(C) 2014 The Japan Society of Plasma Science and Nuclear Fusion Research

Keywords: energetic particle instability, Alfvén mode, integrated simulation, gyrofluid, nonlinear MHD, tokamak

DOI: $10.1585 /$ pfr.9.3403077

\section{Introduction}

Simulation models of nonlinear kinetic modes, such as energetic particle destabilized Alfvén instabilities, are typically limited with respect to the continuous time intervals that can be simulated. Such limitations can arise from a variety of sources: computational resource limits, numerical stability of the underlying time-stepping algorithm, and difficulty in maintaining a balance of the physical drive, damping, and source/sink mechanisms. However, experimental observations [1] have shown that while linear growth e-folding times may be in the range of 50 100 Alfvén times, in the nonlinearly saturated state, these instabilities often persist for times on the order of $10^{5}$ Alfvén times. In order to evaluate the effect of such instabilities on fast ion confinement, it is important to calculate not only their linear thresholds and mode structure, but also the variation in mode structure occurring during these long sustained nonlinear states. It is also necessary to simulate multiple interacting Alfvén modes, since many observations indicate the simultaneous presence of more that one discrete Alfvén frequency line.

In this paper a reduced-dimensionality Landau-fluid closure model for energetic particle driven instabilities is described and applied to a particular case where several Alfvén instabilities are present. This type of model has several features that make it useful for the simulation of long-lived nonlinear states: (a) it is a continuum model so particle noise and associated error accumulation over time is not an issue, (b) it is computationally efficient so longer time interval modeling is feasible, and (c) a variety of source/sink models can be used and nonlinear coupling effects can be turned on and off to test different mechanisms.

The linear version of the model (TAEFL) [2,3] used in this paper has recently been verified against several other gyrokinetic models and validated against experimental RSAE frequency sweep modes [4]. It has also been applied for modeling Alfvén cascade modes [3] using new closure relations that were optimized to represent the Landau response function over a range of phase velocities. Nonlinear versions of TAEFL have been developed [5] by including convective and magnetic flutter nonlinearities in the fluid-like time evolution equations. Although such a model does not explicitly include nonlinear waveparticle trapping effects, the nonlinearities included are basic to both fluid and kinetic instabilities and are sufficient to give saturation via mode coupling effects; mode coupling drives density flattening in the fast ion profile, zonal flows and zonal currents. Techniques for introducing nonlinear wave-particle trapping effects into Landau-fluid models have been outlined and tested [6] for drift wave instabilities and are applicable to the TAEFL model, but are beyond the scope of the current paper.

\footnotetext{
author'se-mail: spongda@ornl.gov

${ }^{*}$ This article is based on the presentation at the 23rd International Toki Conference (ITC23).
} 


\section{Description of the Model and Test Case}

The current version of TAEFL includes an Ohm's law, toroidal component of vorticity, fast ion density and parallel velocity balance equations, and thermal plasma pressure and parallel flow equations. The fast ion parallel velocity equation is closed using a Hammett-Perkins form of closure [7], which introduces the linear Landau resonant behavior necessary for destabilization of shear Alfvén gap modes. The thermal plasma pressure and parallel flow equations introduce the dynamics and compressibility effects needed to include acoustic couplings and geodesic acoustic mode effects. This set of equations has been given in Ref. [3]. As mentioned above, for nonlinear studies, convective and magnetic flutter nonlinearities are introduced. Also, in order to both aid with numerical stability and reflect physical plasma transport and resistivity, finite diffusivities are introduced into each equation at a level of $D=10^{-5} a^{2} / \tau_{\mathrm{A} 0}$, where $\tau_{\mathrm{A} 0}=R_{0} / \mathrm{v}_{\mathrm{A} 0}$. The dynamical variables are represented using Fourier expansions in poloidal and toroidal angles and on a finite difference grid in the toroidal magnetic flux coordinate. For the coupled RSAE/TAE case considered here a mode distribution of $n$ $=0, m=0$ to $10 ; n=2, m=0$ to $20 ; n=3 m=1$ to 21 was used. A new time-stepping algorithm has been implemented that uses dual time steps to make the nonlinear terms more implicit. The governing equations can be written in the form:

$$
\mathbf{L} \frac{\partial \mathbf{y}}{\partial t}=\mathbf{R y}+\mathbb{N}(\mathbf{y})
$$

Here $\mathbf{y}$ is the solution state vector that includes mode amplitudes for the six dynamical variables as a function of flux surface and Fourier $(m, n)$ mode pair.

$$
\begin{aligned}
& \mathbf{L} \frac{\mathbf{y}^{*}-\mathbf{y}^{\prime}}{\Delta t}=\mathbf{R} \frac{\mathbf{y}^{*}-\mathbf{y}^{\prime}}{2}+\mathbb{N}\left(\mathbf{y}^{\prime}\right) \\
& \Rightarrow \mathbf{y}^{*}=\left(\mathbf{L}-\mathbf{R} \frac{\Delta t}{2}\right)^{-1}\left[\left(\mathbf{L}+\mathbf{R} \frac{\Delta t}{2}\right) \mathbf{y}^{\prime}+\Delta t \mathbb{N}\left(\mathbf{y}^{\prime}\right)\right] \\
& \mathbf{L} \frac{\mathbf{y}^{t+\Delta t}-\mathbf{y}^{\prime}}{\Delta t}=\mathbf{R} \frac{\mathbf{y}^{t+\Delta t}+\mathbf{y}^{\prime}}{2}+\frac{1}{2}\left[\mathbb{N}\left(\mathbf{y}^{\prime}\right)+\mathbb{N}\left(\mathbf{y}^{*}\right)\right] \\
& \Rightarrow \mathbf{y}^{t+\Delta t}=\left(\mathbf{L}-\mathbf{R} \frac{\Delta t}{2}\right)^{-1}\left[\left(\mathbf{L}+\mathbf{R} \frac{\Delta t}{2}\right) \mathbf{y}^{\prime}\right. \\
& \left.\quad+\frac{\Delta t}{2}\left(\mathbb{N}\left(\mathbf{y}^{\prime}\right)+\mathbb{N}\left(\mathbf{y}^{*}\right)\right)\right]
\end{aligned}
$$

The above equations show the time discretization using an implicit stepping method for the linear terms (the $\mathbf{L}$ and $\mathbf{R}$ matrix operators) and an explicit dual step predictorcorrector method for the nonlinear terms (the $\mathbb{N}$ matrix operator). This new algorithm has allowed longer time steps to be used in the nonlinear regime, permitting the longer time-scale nonlinear dynamics to be simulated. While a fully implicit stepper might lead to further improvements, this would involve much more extensive changes to the code and involves iterative linear systems solves at each time step, increasing computational costs.
The case to be analyzed here is a noncircular DIIID tokamak reversed shear q-profile $\left(q_{\min }=3.26,\langle\beta\rangle=\right.$ $\left.2 \%, R_{0} /\langle a\rangle=0.42, \kappa=1.67, \delta=0.077\right)$ case similar to that used in Ref. [3], in which an $n=2$ RSAE and an $n$ $=3 \mathrm{TAE}$ are simultaneously unstable. Such a case is a good test for a nonlinear mode-coupling model, such as TAEFL, since having two unstable modes active makes mode coupling effects more dominant. This case also resulted in a linear mode that had up-down asymmetrical (twisting) mode structures. Such asymmetries lead to larger Reynolds and Maxwell stress contributions. i.e., Reynold's stress $\sim\left\langle\mathrm{v}_{\rho} \mathrm{v}_{\theta}\right\rangle$, where $\langle\ldots\rangle$ is a flux surface average. This will be close to zero for up-down symmetrical modes, but non-zero for twisting, up-down asymmetrical modes.

\section{Nonlinear RSAE/TAE Simulation Results}

The above case has been run using the TAEFL model for approximately $10^{4} \tau_{\mathrm{A} 0}$. In order to sustain an Alfvén mode over these time scales and to study its self-regulation via zonal flows and currents, the fast ion density profile is kept fixed in time.

Since the nonlinear couplings would normally flatten the density near where the mode is dominant, this implies the presence of a particle source that continuously fills in the profile. Such a model is implemented simply by not including the feedback of the nonlinearly generated $m, n$ $=(0,0)$ component in the fast ion density evolution equation. In Fig. 1 the resulting edge magnetic fluctuations vs. time for this run are shown. In Fig. 1 (a) the contours of the poloidal velocity are plotted vs. Rho $=$ (normalized toroidal flux $)^{1 / 2}$ and time, in (b) the edge magnetic fluctuations vs. time are given, and in (c) the spectrogram of these fluctuations is given. As indicated, after passing through a linear growth phase, the amplitude of the fluctuations peaks, decays over the interval $t / \tau_{\mathrm{A} 0}=3500$ to about 5000 , then begins increasing again, peaks out at about $t / \tau_{\mathrm{A} 0}=5300$, decays again, and so on. These growth/decay cycles repeat until the calculation is stopped at around $t / \tau_{\mathrm{A} 0}=10^{4}$. The peaks in the fluctuations correlate with times of high radial shearing in the poloidal (zonal) velocity, as indicated by radial bunching of the contours in Fig. 1 (a). The spectrogram shows that initially two discrete frequency peaks are present. These couple and produce frequency splittings. Low frequency components are also generated when the mode amplitude enters its decaying phases. These low frequency bands are associated with the zonal flows and currents that are being generated through the Reynold's and Maxwell stress effects; due to their nonlinear origin, they do not reflect the same Alfvén frequency time variation as the unstable modes.

In Fig. 2 the time and radial variationof the poloidalcomponent of $\boldsymbol{E} \times \boldsymbol{B}$ velocity is plotted on the vertical axis. The peaks in this zonal flow velocity correlate well in time 

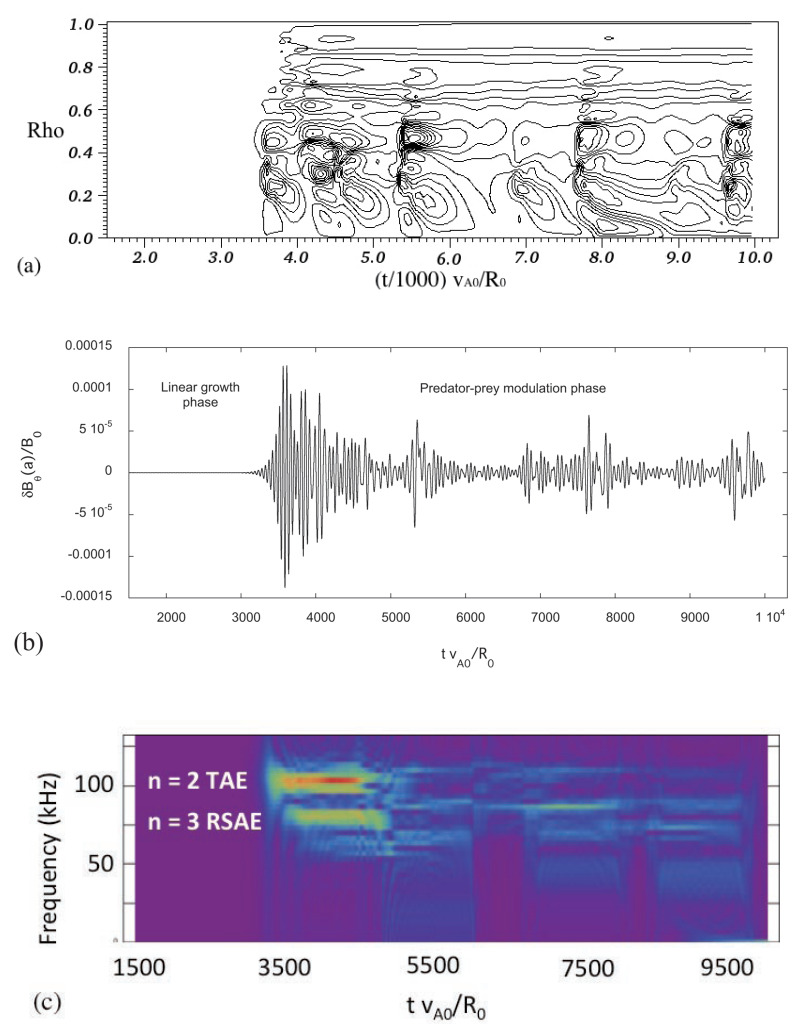

Fig. 1 (a) Contours of zonal flow velocity vs. $\rho$ and time, (b) Predicted edge magnetic fluctuations from Alfvén instabilities, (c) Spectrogram of data from (b).

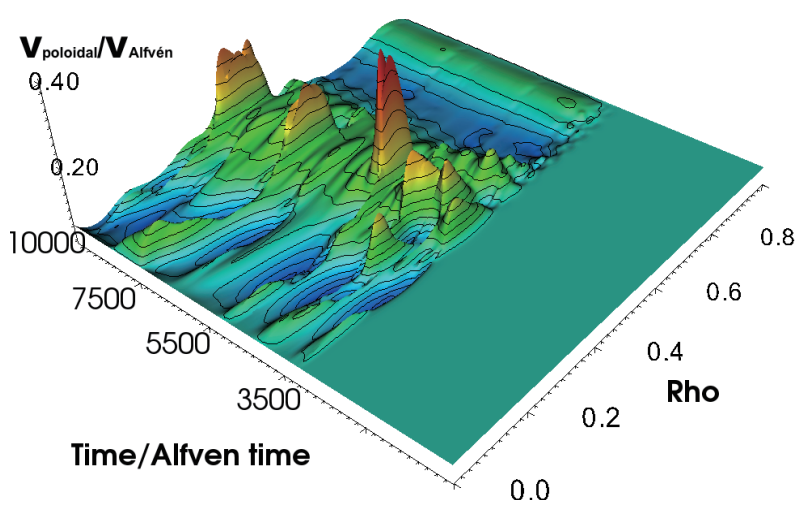

Fig. 2 Time evolution of nonlinearly driven poloidal flow velocity, indicating strong shearing as AE mode amplitude peaks.

with the peaks in the edge magnetic fluctuations shown in Fig. 1 (a). The role of zonal flows and currents in the saturation of Alfvén instabilities have recently been emphasized in several analytical models [8,9]. Also, simulations have examined the effects of driven zonal flows on nonlinear TAE saturation [10]. In Fig. 3 the mode structure of the electrostatic potential function is shown on a constant toroidal angle plane at 4 times during the simulation. At $t=2000 \tau_{\mathrm{A} 0}$, the instability is in its linear growth phase. At $t=3600 \tau_{\mathrm{A} 0}$, the mode is at it's peak amplitude and is
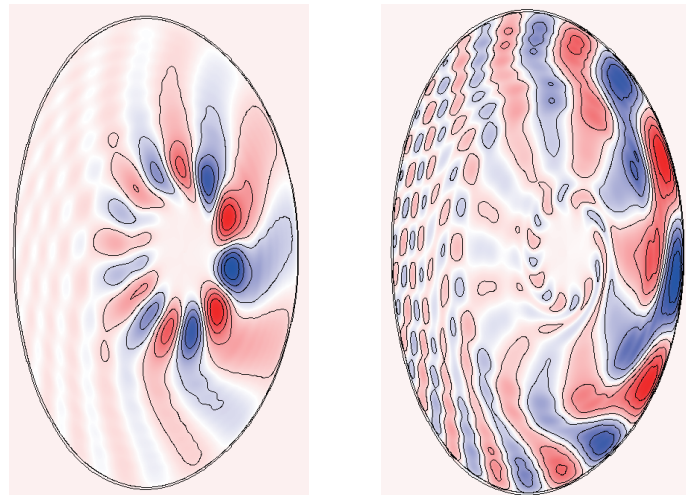

$\mathrm{t}=2000 \tau_{\mathrm{A} 0}$

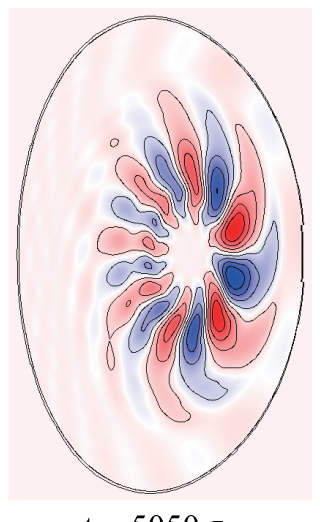

$\mathrm{t}=5050 \tau_{\mathrm{A} 0}$

$$
\mathrm{t}=3600 \tau_{\mathrm{A} 0}
$$

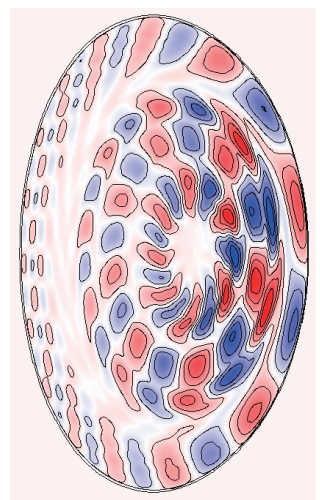

$\mathrm{t}=5300 \tau_{\mathrm{A} 0}$
Fig. 3 Mode structure evolution, showing alternating variation from coherent linear mode structure $(2000,5050)$ and sheared radially expanded structure $(3600,5300)$.

driving strong zonal flows and currents, which are shearing the mode structure and causing it to expand radially. This radial expansion is related to the fact that maximum zonal flow shearing (radial gradient of the velocity in Fig. 2) occurs to the inside and outside of the peak in the linear mode structure.

Also, the $n=2$ TAE mode (which the $n=3$ RSAE couples with) is localized further out radially than the $n=$ $3 \mathrm{RSAE}$ mode. At $t=5050 \tau_{\mathrm{A} 0}$, the zonal flows/currents have suppressed the amplitude enough that the mode structure is essentially in back its linear regime again. At $t$ $=5300 \tau_{\mathrm{A} 0}$, the amplitude has increased and strong zonal flows/currents are again active and shearing the mode structure. This predator-prey sequence between growing mode amplitude and suppression by zonal flow/current shearing repeats itself for as long as the simulation is run.

\section{Conclusions}

A nonlinear simulation of a coupled $n=2$ TAE and $n=3$ RSAE instability has been carried out using the TAEFL Landau-fluid model. The focus of this effort has been an investigation of issues involved in simulating the very long-lived coherent Alfvén frequency spectral lines 
that are often observed experimentally in reversed shear qprofile discharges. These issues include both the numerical stability of the time-stepping algorithm as well as balancing the various nonlinear saturation mechanisms so that the turbulent state becomes self-regulating. A minimalist model is used based on constructing a fast ion particle source, which exactly balances the nonlinearly driven profile flattening. This facilitates a study of the dynamics of zonal flows and currents that are driven by mode coupling effects. Predator-prey dynamics are found to regulate the nonlinear state and have been followed for on the order of $10^{4}$ Alfvén times. Mode coupling effects for energetic particle-destabilized Alfvén modes are likely to become increasingly important in future large ignited tokamaks, such as ITER, where the most unstable toroidal mode number shifts to higher values (due the decreasing value of $\rho_{* \mathrm{EP}}$ ) and a larger range of adjacent toroidal mode numbers will be simultaneously unstable. Evaluating the nonlinear dynamics of strongly mode coupled Alfvén instablilities in this regime will be critical to predictions of the losses of alpha particles and other fast ion components.

\section{Acknowledgements}

This work is supported both by the US Department of Energy under Contract DE-AC05-00OR22725 with UT-
Battelle, LLC and under the US DOE SciDAC GSEP Center. These calculations have been motivated by the extensive experimental work of Ben Tobias, Mike Van Zeeland, and William Heidbrink. Also, many useful interactions are acknowledged with members of the GSEP SciDAC project (Zhihong Lin, Eric Bass and Wenjun Deng).

[1] B.J. Tobias, I.G. Classen, C.W. Domier, W.W. Heidbrink, N.C. Luhmann, R. Nazikian, H.K. Park, D.A. Spong and M.A. Van Zeeland, Phys. Rev. Lett. 106, 075003 (2011).

[2] D.A. Spong, B.A. Carreras and C.L. Hedrick, Phys. Fluids B4, 3316 (1992).

[3] D.A. Spong, Nucl. Fusion 53, 053008 (2013).

[4] D.A. Spong, E.M. Bass, W. Deng, W.W. Heidbrink, Z. Lin, B. Tobias, M.A. Van Zeeland, M.E. Austin, C.W. Domier and N.C. Luhmann, Jr., Phys. Plasmas 19, 0825111 (2012).

[5] D.A. Spong, B.A. Carreras and C.L. Hedrick, Phys. Plasmas 1, 1503 (1994).

[6] N. Mattor, Phys. Rev. Lett. 79, 3419 (1997).

[7] G.W. Hammett and F.W. Perkins, Phys. Rev. Lett. 64, 3019 (1990).

[8] L. Chen and F. Zonca, Phys. Rev. Lett. 109, 145002 (2012).

[9] L. Chen and F. Zonca, Phys. Plasmas 20, 055402 (2013).

[10] Y. Todo, H.L. Berk and B.N. Breizman, Nucl. Fusion 50, 084016 (2010). 